\title{
Survival and normal function of glycolysis-deficient mouse oocytes
}

\author{
A. Kelly* and J. D. West ${ }^{\dagger}$ \\ Genes and Development Group, Department of Reproductive and Developmental Sciences, \\ University of Edinburgh, Hugh Robson Building, George Square, Edinburgh EH8 9XD, UK
}

\begin{abstract}
Mouse embryos homozygous for a null allele of Gpi1 which encodes the glycolytic enzyme glucose phosphate isomerase fail to complete gastrulation and die at about embryonic day 7.5, but mutant cells can survive in fetal chimaeras in which they are mixed with wild-type cells. An adult female mouse chimaera, composed of wild-type cells and homozygous Gpi1/- null mutant cells, was produced to test whether the presence of wild-type cells in the ovary allowed mutant oocytes to survive and function. This mouse produced 28 offspring, eight of which were derived from homozygous $\mathrm{Gpi1}^{-/}$null oocytes. DNA in situ hybridization also showed that some $\mathrm{Gpi}^{-/-}$follicle cells
\end{abstract}

were able to survive in chimaeric ovarian follicles. It is likely that the survival of mutant follicle cells and fully functional mutant oocytes was mediated by the presence of wild-type cells that could provide metabolic intermediates and so bypass the block in the glycolytic pathway. Wild-type cumulus cells probably supported the growing GPI-deficient oocytes via metabolic co-operation, by passing ATP and other glycolytic products through gap junctions. It was concluded that female mouse germ cells and ovarian follicle cells do not need an intact endogenous glycolytic pathway if they can obtain appropriate metabolites from an exogenous source.

\section{Introduction}

Mouse embryos homozygous for a null mutation (Gpi1-sa-m1H, abbreviated to Gpi ${ }^{-}$) in the gene encoding the glycolytic enzyme, glucose phosphate isomerase (GPI; E.C. 5.3.1.9) die early in development (West et al., 1990) and fail to complete gastrulation (Kelly and West, 1996). Although the homozygous Gpi1- null genotype is lethal to embryos it need not be lethal to cells. Homozygous Gpi1- null cells co-existing with wild-type Gpi1 cells in chimaeric Gpi $1^{--} \leftrightarrow G p i 1^{c / c}$ conceptuses survive, usually in small numbers, to at least embryonic day 12.5 and have the developmental potential to contribute to a wide range of embryonic and extraembryonic tissues (Kelly and West, 2002). The aim of the present study was to determine whether Gpi $1^{-/}$null cells can survive in Gipi-1 $\leftrightarrow G p i 1^{c / c}$ chimaeras until adulthood and whether homozygous Gpi $1^{-/}$null female germ cells can produce fully functional oocytes.

\section{Materials and Methods}

Mice

The Gpi1-sa-m1H mutant null allele (abbreviated to Gpi1-) was maintained by mating Gpi $1^{a /-} \times G p i 1^{b / b}$ and Gpi $1^{b /-} \times$

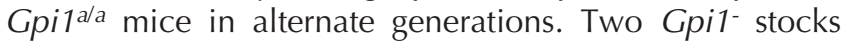
('GN' and 'NUL') were used in the present study as

*Present address: Genetics Division, University of Nottingham, Medical School, Queens Medical Centre, Nottingham NG7 2UH, UK ${ }^{+}$Correspondence

Email: John.West@ed.ac.uk described by Kelly and West (2002). Stock 'GN' was pigmented and Gipit/-, and stock 'NUL' was pigmented, Gpi1//- and homozygous $\left(\mathrm{Tg}^{+/+}\right)$for the reiterated $\beta$-globin transgene TgN(Hbb-b1)83Clo (Lo, 1986; Lo et al., 1987).

\section{Production of chimaeras}

Chimaeras were produced by aggregating pairs of preimplantation embryos produced from superovulated females using the method developed by Tarkowski (1961), as fully described by West and Flockhart (1994). Eight-cell stage, genetically pigmented embryos from $\mathrm{Gpi}^{\mathrm{bl}-}, \mathrm{Tg}^{-1-} \times$ $\mathrm{Gpi}^{2 /-}, \mathrm{Tg}^{+/+}(\mathrm{GN} \times \mathrm{NUL})$ crosses were flushed from the uterus at embryonic day 2.5 and aggregated with eightcell stage, genetically albino Gpi $1^{c / c}$ embryos (designated ' $\mathrm{CF}_{2}{ }_{2}$ ) produced by intercrossing (C57BL-Gpi1', C/Ws $\times$ BALB/c-Gpi1'/Ws) $\mathrm{F}_{1}$ hybrids (' $\mathrm{CF}_{1}$ ' hybrids). After culture overnight, the embryonic day 3.5 chimaeric aggregates were transferred to the uteri of pseudopregnant ' $\mathrm{CF}_{1}$ ' (homozygous Gpi1d/c) females 2.5 days after mating to vasectomized males and allowed to go to term. This procedure resulted in pigmented $\leftrightarrow$ albino chimaeras of four genotype combinations: Gpi $1^{a / b} \leftrightarrow G p i 1^{c / c}, G p i 1^{a /-} \leftrightarrow G p i 1^{d c}$,

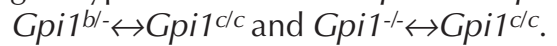

\section{Analysis of chimaeras}

The percentage of pigment in the coats and eyes of adult chimaeras was estimated subjectively. Mice were killed by exposure to carbon dioxide and their eyes were removed and examined under a dissecting microscope for overall pigmentation in the superimposed choroid and retinal 

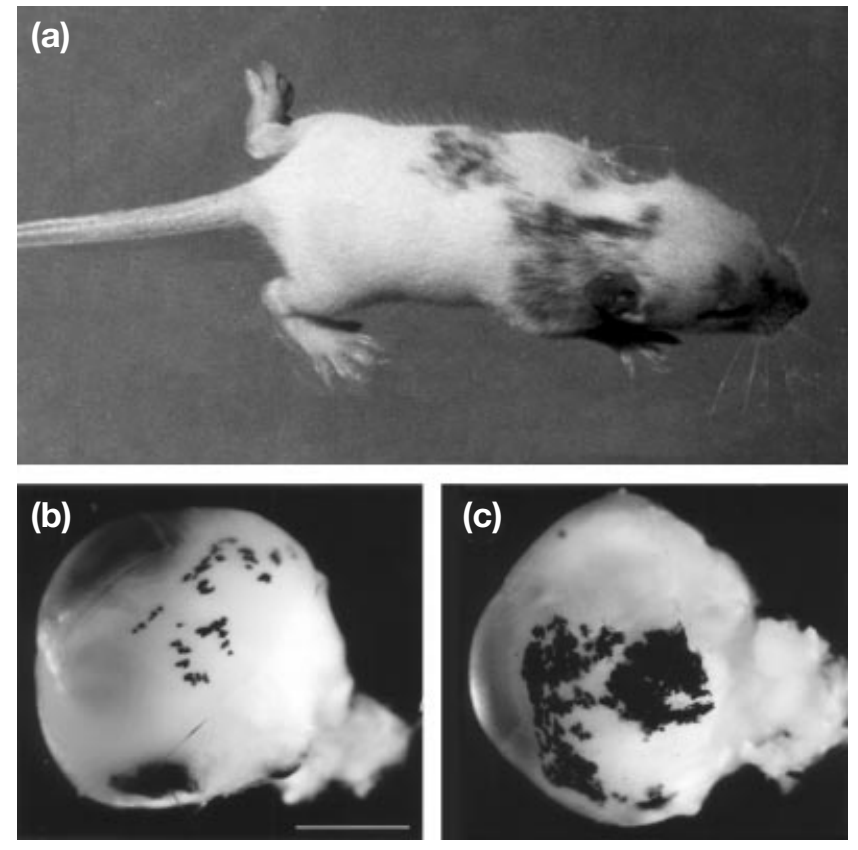

Fig. 1. Homozygous Gpi1- $\leftrightarrow G$ Gi1 ${ }^{c / c}$ chimaera AK2. (a) Chimaera AK2 at 2 weeks. (b,c) Two views of the right eye. Scale bar represents $1 \mathrm{~mm}$.

pigment epithelium. Various organs were removed and samples were prepared for analysis by GPI electrophoresis and DNA in situ hybridization. Cellulose acetate electrophoresis of GPI and quantitative densitometry were performed as described by West and Flockhart (1994). A small blood sample was taken from the tail vein of anaesthetized live mice at 5 weeks for GPI analysis and to prepare blood smears for DNA in situ hybridization. Smears were air dried on clean microscope slides, fixed in ethanol: acetic acid (3:1) for $60 \mathrm{~min}$, air dried, immersed in acetone for $10 \mathrm{~min}$ and dehydrated through a graded series of alcohol before in situ hybridization.

DNA in situ hybridization to the reiterated $\beta$-globin transgene $\mathrm{TgN}(\mathrm{Hbb}-\mathrm{b} 1) 83 \mathrm{Clo}$ was detected in tissue sections and blood smears by diaminobenzidine staining for a peroxidase-conjugated antibody bound to the digoxygeninlabelled DNA probe (Keighren and West, 1993). Slides were counterstained with haematoxylin and eosin, and examined by bright-field microscopy to identify $\mathrm{Tg}^{+/-}$cells derived from the GN $\times$ NUL component in the chimaera by the presence of a brown spot (hybridization signal) in the nucleus. For each tissue section or blood smear, approximately 300 nuclei were scored for the presence of the hybridization signal.

\section{Results}

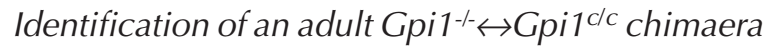

Eleven live mice were born after embryo aggregation and nine were identified as chimaeras by coat colour variegation
(Fig. 1a). GPI electrophoresis of peripheral blood identified

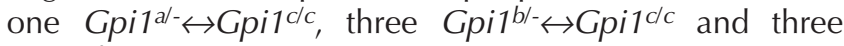
$G i 1^{a / b} \leftrightarrow G p i 1^{c / c}$ chimaeras. The remaining two chimaeras (females AK1 and AK2) each had some coat pigment but their blood samples produced only GPI1-C activity. It is possible that these were $\mathrm{Gpi}^{-{ }^{-} \leftrightarrow G \mathrm{Gi}} 1^{c / c}$ chimaeras but it is difficult to distinguish Gpi $1^{-} \leftrightarrow G$ Gi1 ${ }^{c / c}$ chimaeras from other chimaeras with a very high proportion of $\mathrm{Gpi}^{\mathrm{c} / c}$ cells.

Electrophoresis of mixtures of GPI1-B and GPI1-C tissue homogenates showed that as little as 3\% GPI1-B (by tissue mass) was consistently detected (Kelly, 1995). Thus, $(\mathrm{GN} \times \mathrm{NUL})$ cells should be detectable in blood samples from chimaeras by GPI1 electrophoresis, if they contributed at least $3 \%$ to the blood. The composition of different tissues in individual chimaeras tends to be similar (Falconer et al., $1981)$, so usually $(\mathrm{GN} \times \mathrm{NUL})$ cells should be detectable in the blood if they contribute significantly to other tissues. Chimaera AK1 had $<1 \%$ pigment in the coat and eyes, so its genotype remains unknown. It could have been either Gpi $1^{-} \leftrightarrow G$ Gi $11^{c / c}$ or another chimaeric genotype with undetectable concentrations of GPI1-A, -B or -AB in the blood. However, the coat of chimaera AK2 contained a significant amount of pigment (Fig. 1a) and was classified as $10 \%$ pigmented in the adult. Therefore, AK2 was provisionally classified as a Gpi $1^{--} \leftrightarrow G \mathrm{Gi} 1^{c / c}$ chimaera, implying that homozygous Gpi 1 - null coat melanocytes can survive to adulthood.

DNA in situ hybridization was performed on blood smears from chimaera AK2 at 5 weeks and 31/399 (8\%) nucleated blood cells carried the transgenic marker. The presence of $8 \%(G N \times N U L)$ cells in the absence of $(\mathrm{GN} \times \mathrm{NUL}) \mathrm{GPI}$ activity provides more direct evidence for the conclusion that AK2 is a genuine Gpi $1^{-} \leftrightarrow G p i 1^{c / c}$ chimaera. This finding also demonstrates that homozygous Gpi1-- leucocytes survived for at least 5 weeks.

\section{Homozygous Gpi 1- null germ cells can produce viable and functional oocytes}

Chimaera AK2 was mated to an albino Gpi $1^{c / c}$ male (stock $\mathrm{CF}_{1}$ ) to confirm that it was a Gi1 ${ }^{-r_{\leftrightarrow}} \leftrightarrow \mathrm{Gpi}^{\mathrm{c} / \mathrm{c}}$ chimaera and to test whether gametes derived from homozygous Gpi $1^{-1-}$ mutant germ cells were functional. The progeny were classified for coat colour and GPI phenotype. Both Gpi1c/c and Gpi1cl- offspring produced a single GPI1-C band by electrophoresis but the genotypes of the GPI1-C offspring could be deduced without test breeding (for example, GPI1-C offspring from the Gpi $1^{b /-}$ component of a pigmented Gpi ${ }^{b /-} \leftrightarrow$ albino Gpi $1^{c / c}$ chimaera mated to a Gpi $1^{c / c}$ mouse would be pigmented and Gpi1/-, whereas those from the Gpi $1^{c / c}$ component would be albino and Gpitclc).

Chimaera AK2 produced 28 offspring, 20 of which were albino and GPI1-C $\left(G p i 1^{c / c}\right)$, and eight of which were pigmented and GPI1-C (Gpi $\left.1^{c /}\right)$. The eight pigmented offspring were distributed among all three litters which yielded 2/8, $3 / 8$ and 3/12 pigmented offspring, respectively. Pigmented 
Gpi $1^{c-}$ offspring could be produced from matings with albino Gpi 1/c males if the pigmented component of the

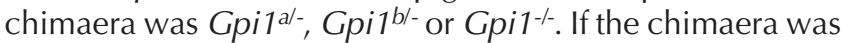
either Gpi ${ }^{a /-} \leftrightarrow G p i 1^{c / c}$ or Gpi $1^{b /-} \leftrightarrow G p i 1^{c / c}, 50 \%$ of the pigmented offspring should be Gpi a/c or Gpi $1^{b / c}$, whereas if it was Gpi ${ }^{-} \leftrightarrow G$ Gi $1^{c / c}$ all of the pigmented offspring should be GPI1-C (Gpi $\left.{ }^{c /}\right)$. The probability that all eight pigmented offspring were GPI1-C if the chimaera was either Gpi $1^{a /} \leftrightarrow G p i 1^{c / c}$ or $G p i 1^{b /-} \leftrightarrow G p i 1^{c / c}$ is only $(0.5)^{8}$ (that is, $P=0.004$ ), which supports the conclusion that AK2 is a Gpi $1^{-} \leftrightarrow G p i 1^{1 / c}$ chimaera.

The genotypes of albino and pigmented GPI1-C offspring of the mating between chimaera AK2 and the albino Gpi 1c/c male were confirmed by test-mating some of the offspring to pigmented Gipi ${ }^{b / b}(\mathrm{C} 57 \mathrm{BL} / \mathrm{Ws} \times \mathrm{CBA}) \mathrm{F}_{1}$ hybrid mice. Four albino GPI1-C offspring (putative Gpi1c/c genotype) each produced only GPI1-BC (Gpi $1^{\text {b/c }}$ ) progeny (90 in total), as expected for a Gpi1c/c $\times G p i 1^{b / b}$ cross. Five of the eight pigmented GPI1-C offspring (putative Gpi ${ }^{c /}$ - genotype) were similarly test-mated and all five produced some GPI1B (Gpi $\left.1^{b /-}\right)$ and some GPI1-BC (Gpi $\left.1^{b / c}\right)$ progeny (66 GPI1-B and 66 GPI1-BC in total), as expected for a Gpi $1^{c-} \times G p i 1^{b / b}$ cross. This finding confirms that all the AK2 pigmented GPI1-C progeny that were test-mated were Gpi1c/- rather than Gpi $1^{1 / c}$. The results of the genetic test-crosses confirm that AK2 is a genuine Gpi $1^{--\leftrightarrow} \leftrightarrow$ Gpi $11^{c / c}$ chimaera and demonstrate that homozygous Gi1 $1^{--}$null oogonia can produce viable and functional oocytes that result in viable offspring when fertilized with wild-type spermatozoa.

\section{GPI analysis of other tissues}

After completion of the test breeding, the chimaeras were killed and GPI electrophoresis was performed on $>20$ tissue samples. Chimaera AK2 died while giving birth (aged 21 weeks), but a full set of samples was analysed successfully for GPI. All samples were entirely GPI1-C (in agreement with the 5 week blood analysis), which is consistent with the conclusion that this mouse was a genuine Gpi $1^{-1} \leftrightarrow G$ Gi $1^{c / c}$ chimaera.

\section{Tissue composition of Gpi $1-1 \leftrightarrow G p i 1^{c / c}$ chimaera AK2}

After chimaera AK2 died, its eyes were removed and the percentage of eye pigment was estimated as approximately $15 \%$. Although this is a crude estimate because variegation in the retinal pigment epithelium may be obscured by pigment in the overlying choroid, pigmented homozygous Gpi1-- null cells survived at least in the choroid of Gpi $1^{-1} \leftrightarrow G$ Gi $1^{c / c}$ chimaera AK2 until 21 weeks (Fig. 1b,c).

Although chimaera AK2 died, DNA in situ hybridization was performed successfully on a range of tissues and the transgenic lineage marker, present in the Gpi1-/- null cells, was detected in the right ovary, brain, uterus, liver, thymus, small intestine and bladder. Generally the number of mutant cells was small but one ovarian follicle with a mutant oocyte and a high proportion of Gpi1-/- follicle cells was found (Fig. 2). Gpi 1-/- null cells were not detected by in

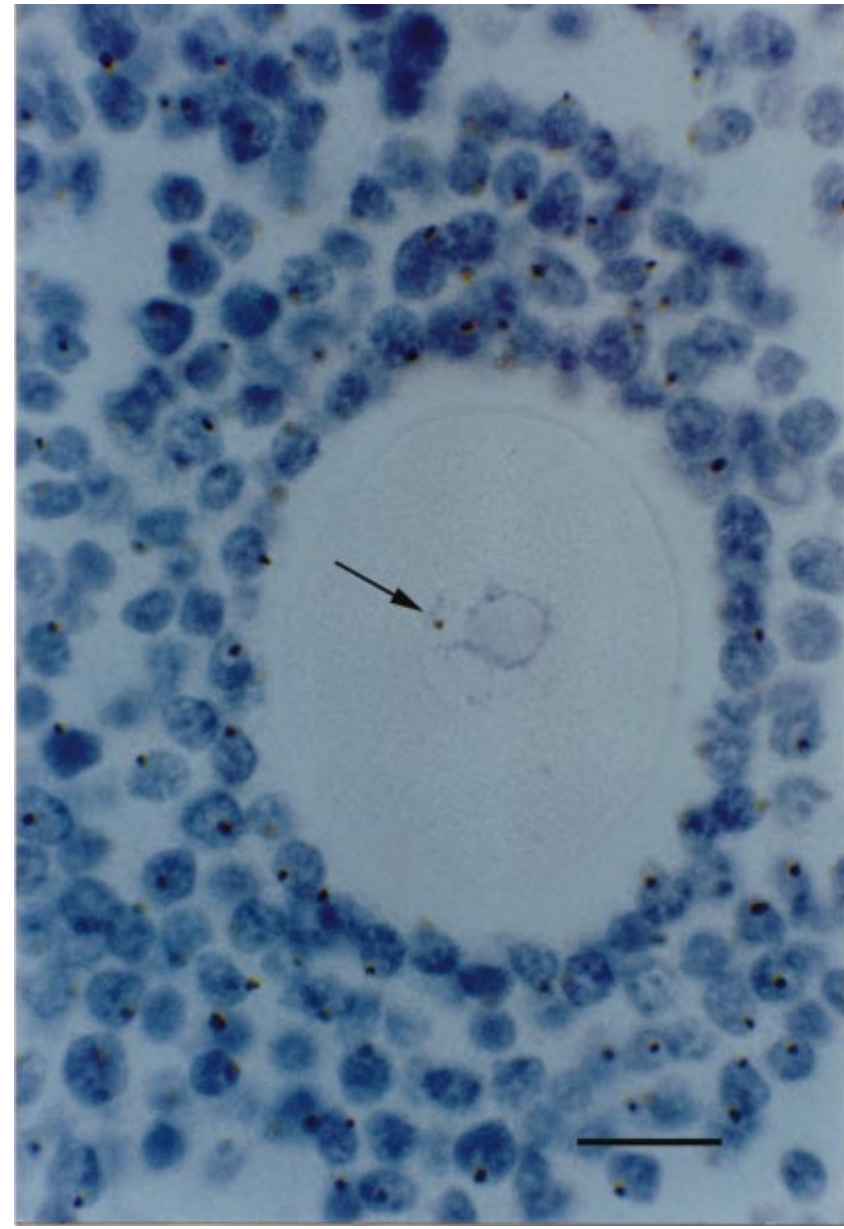

Fig. 2. Ovarian follicle from Gpi1 ${ }^{-} \leftrightarrow G p i 1^{c / c}$ chimera AK2 after DNA in situ hybridization to detect the reiterated $\beta$-globin transgene in $\mathrm{Gpi1}^{-/}$cells, which were hemizygous $\left(\mathrm{Tg}^{+/-}\right)$for the transgene. Hybridization to the transgene (visualized as a brown spot in the nucleus) can be seen in the oocyte (arrow) and in many of the surrounding follicle cells, indicating that they are mutant Gpi $1^{-1}$ cells. Other follicle cells are wild type and lack the brown hybridization signal. Scale bar represents $20 \mu \mathrm{m}$.

situ hybridization in heart, right kidney, pancreas, spleen, lung, large intestine and caecum plus appendix, but it is not clear whether this indicates that mutant cells were excluded from these tissues or simply reflects the poor preservation of the tissues from the dead mouse. For this reason no attempt was made to quantify the Gpi1-1 contribution to the other tissues.

\section{Discussion}

The results of the present study demonstrate that one adult female mouse (AK2) was a genuine Gpi1- $\leftrightarrow$ Gpi1 ${ }^{c / c}$ chimaera and that some homozygous Gpi $1^{-/}$null cells can survive in a range of adult tissues, including coat melanocytes, choroidal melanocytes, leucocytes, brain, uterus, liver, thymus, small intestine, bladder, ovarian follicle cells 
and oocytes. Homozygous Gpi $1^{-1}$ cells would be unable to generate energy via glycolysis, unless wild-type cells provided substrates downstream of GPI, but they could produce energy by the tricarboxylic acid cycle (TCA cycle or Krebs cycle) if appropriate substrates (for example, pyruvate or glutamine) were available. The low survival seen in several fetal Gipi-1 $\leftrightarrow G$ Gi1 ${ }^{c / c}$ chimaeras reported by Kelly and West (2002) indicates that most Gpi $1^{-1-}$ cells are at a selective disadvantage and need an intact glycolytic pathway to function optimally.

The in situ hybridization analysis revealed that mutant follicle cells and oocytes can survive in chimaeric ovarian follicles composed of a mixture of mutant and wild-type cells. The genetic crosses demonstrate that such oocytes can be ovulated and fertilized, and will produce normal fertile offspring.

Mouse oocytes use pyruvate rather than glucose as a major energy source, whereas follicle cells actively metabolize glucose to pyruvate and rely predominantly on glycolysis for energy provision between mid-preantral and preovulatory stages (Biggers et al., 1967; Donahue and Stern, 1968; Boland et al., 1994; Downs and Utecht, 1999). Pyruvate is secreted by cultured follicle cells (Donahue and Stern, 1968; Leese and Barton, 1985; Downs and Mastropolo, 1994) and if this occurs in vivo pyruvate may diffuse through the intercellular space and be available to rescue Gpi $1^{--}$null follicle cells and oocytes. Downs et al. (2002) reported that most of the ATP used by oocytes in cultured oocyte-cumulus complexes is generated by glycolysis (in follicle cells) rather than from oxidation of pyruvate. Follicle cells communicate with each other and with oocytes via gap junctions (Anderson and Albertini, 1976; Gilula et al., 1978) and probably pass metabolites, such as glucose-6-phosphate (Saito et al., 1994) and ATP (Downs and Mastropolo, 1994; Downs, 1995), to the oocytes via the gap junctions.

Mutant Gpi $11^{-/}$null oocytes could produce energy by metabolizing pyruvate in the TCA cycle, so that surrounding wild-type follicle cells could rescue the oocytes by providing either ATP or pyruvate. Pyruvate could diffuse via the intercellular space, and both pyruvate and ATP could be transferred to mutant oocytes via gap junctions and so rescue them by metabolic co-operation. Mutant GPIdeficient follicle cells could produce energy by glycolysis downstream of the blocked GPI step that converts glucose6-phosphate to fructose-6-phosphate. Thus, transfer of ATP or glycolytic products downstream of glucose-6-phosphate through gap junctions would allow wild-type follicle cells to rescue neighbouring Gpi $1^{--}$null follicle cells by metabolic co-operation.

It also follows that mutant Gpi $1^{-/}$primordial germ cells and oogonia were able to survive in the chimaera. It is likely that these earlier stage mutant germ cells were also dependent on metabolites produced by wild-type cells in the chimaera. Survival of embryos produced by fertilization of null oocytes with wild-type spermatozoa can also be explained because preimplantation mouse embryos do not use glucose as an energy source until the eight-cell stage (Brinster, 1965; Gardner and Leese, 1986), which is about the time that the wild-type paternal Gpi ${ }^{+}$allele would be activated, thereby restoring an intact glycolytic pathway (Chapman et al., 1971; Brinster, 1973; West and Green, 1983; Duboule and Burki, 1985; Gilbert and Solter, 1985; West et al., 1986).

By a combination of DNA in situ hybridization and genetics the present study has shown that oocytes from homo-

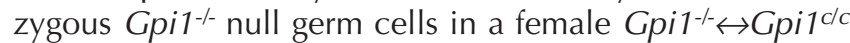
chimaera are both viable and functional, and that mutant ovarian follicle cells can also differentiate and survive. This finding demonstrates that neither female mouse germ cells nor follicle cells need an intact endogenous glycolytic pathway provided that they can obtain appropriate exogenous metabolites.

The authors thank D. Doogan, M. Ross and J. Macdonald for expert mouse husbandry, N. Spears for helpful comments, and M. Keighren and T. McFetters for help in preparing the figures. The authors are also grateful to the Wellcome Trust for financial support (grant 046359 to J. D. West).

\section{References}

Anderson E and Albertini DF (1976) Gap junctions between oocyte and companion follicle cells in mammalian ovary Journal of Cell Biology $\mathbf{7 1}$ 680-686

Biggers JD, Whittingham DG and Donahue RP (1967) The pattern of energy metabolism in the mouse oocyte and zygote Proceedings National Academy of Sciences USA 58 560-567

Boland NI, Humpherson PG, Leese HJ and Gosden RG (1994) Characterization of follicular energy metabolism Human Reproduction 9 604-609

Brinster RL (1965) Studies on the development of mouse embryos in vitro. II. The effect of energy source Journal of Experimental Zoology 158 59-63

Brinster RL (1973) Parental glucose phosphate isomerase activity in threeday mouse embryos Biochemical Genetics 9 187-191

Chapman VM, Whitten WR and Ruddle FH (1971) Expression of paternal glucose phosphate isomerase-1 (Gpi-1) in preimplantation stages of mouse embryos Developmental Biology 26 153-158

Donahue RP and Stern S (1968) Follicular cell support of oocyte maturation: production of pyruvate in vitro. Journal of Reproduction and Fertility 17 395-398

Downs SM (1995) The influence of glucose, cumulus cells and metabolic coupling on ATP levels and meiotic control in the isolated mouse oocyte Developmental Biology 167 502-512

Downs SM and Mastropolo AM (1994) The participation of energy substrates in the control of meiotic maturation in murine oocytes Developmental Biology $\mathbf{1 6 2}$ 154-168

Downs SM and Utecht AM (1999) Metabolism of radiolabeled glucose by mouse oocytes and oocyte-cumulus cell complexes Biology of Reproduction 60 1446-1452

Downs SM, Humpherson PG and Leese HJ (2002) Pyruvate utilization by mouse oocytes is influenced by meiotic status and the cumulus oophorus Molecular Reproduction and Development 62 113-123

Duboule D and Burki K (1985) A fine analysis of glucose phosphate isomerase patterns in single preimplantation mouse embryos Differentiation 29 25-28

Falconer DS, Gauld IK, Roberts RC and Williams DA (1981) The control of body size in mouse chimaeras Genetical Research 38 25-46

Gardner DK and Leese HJ (1986) Non-invasive measurement of nutrientuptake by single cultured preimplantation mouse embryos Human Reproduction 125-27 
Gilbert SF and Solter D (1985) Onset of paternal and maternal Gpi-1 expression in preimplantation mouse embryos Developmental Biology $109515-517$

Gilula NB, Epstein ML and Beers WH (1978) Cell-to-cell communication and ovulation. A study of the cumulus-oocyte complex Journal of Cell Biology 78 58-75

Keighren M and West JD (1993) Analysis of cell ploidy in histological sections of mouse tissues by DNA-DNA in situ hybridization with digoxygenin labelled probes Histochemical Journal 25 30-44

Kelly A (1995) The Developmental Potential of Embryos and Cells that are Deficient in Glycolysis PhD Thesis, University of Edinburgh pp 250

Kelly A and West JD (1996) Genetic evidence that glycolysis is necessary for gastrulation in the mouse Developmental Dynamics 207 300-308

Kelly A and West JD (2002) Developmental potential and survival of glycolysis-deficient cells in fetal mouse chimeras Genesis 33 29-39

Leese HJ and Barton AM (1985) Production of pyruvate by isolated mouse cumulus cells Journal of Experimental Zoology 234 231-236

Lo C (1986) Localization of low abundance DNA sequences in tissue sections by in situ hybridization Journal of Cell Science 81 143-162

Lo CW, Coulling M and Kirby C (1987) Tracking of mouse cell lineage using microinjected DNA sequences: analysis using genomic Southern blotting and tissue-section in situ hybridizations Differentiation 35 37-44

Saito T, Hiroi M and Kato T (1994) Development of glucose utilization studied in single oocytes and preimplantation embryos from mice Biology of Reproduction 50 266-270

Tarkowski AK (1961) Mouse chimaeras developed from fused eggs Nature $190857-860$

West JD and Flockhart JH (1994) Genotypically unbalanced diploid'diploid foetal mouse chimaeras: possible relevance to human confined mosaicism Genetical Research 63 87-99

West JD and Green JF (1983) The transition from oocyte-coded to embryocoded glucose phosphate isomerase in the early mouse embryo Journal of Embryology and Experimental Morphology 78 127-140

West JD, Leask R and Green JF (1986) Quantification of the transition from oocyte-coded to embryo-coded glucose phosphate isomerase in mouse embryos Journal of Embryology and Experimental Morphology 97 225-237

West JD, Flockhart JH, Peters J and Ball ST (1990) Death of mouse embryos that lack a functional gene for glucose phosphate isomerase Genetical Research 56 223-236

Received 4 April 2002.

First decision 31 May 2002.

Revised manuscript received 26 June 2002.

Accepted 26 June 2002. 